denied by the majority of biologists, but a vigorous school of thinkers, finding their inspiration rather in philosophy than chemistry, are working to reconcile progressive work in biochemistry with the study of life in the concrete. There are indeed signs that the rest of the century may come to justify the glowing prediction of the late Prof. Patrick Geddes, that it would see the triumph of life and be the age of biology. It is already true, in the more general sense, that men accept as a philosophical idea the community of all life and the development of higher forms of life from lower by some process in time which we have still to unravel.

It would be well if one could speak of the acceptance of a community of human life on the planet with as much confidence as the growing consensus of opinion as to its origin. In this matter, while the King's reign is distinguished by the establishment of the League of Nations, it cannot be said that the idea which it embodies or the practices which it exists to promote have made commensurate progress with what we have had to record of the progress of science. In some respects, there has been in recent years an actual setback. Germany and Japan have renounced the League, and the United States, though helpful and friendly, has not formally joined even the Court of International Justice at The Hague. Armaments have lately increased and no effective grouping or control of aviation has yet been effected. There could be no more flagrant instance of the contrast between the unity of thought, which has given man his command of Nature, and the want of unity in his application of it, than this wanton rivalry in military aviation. The most highly scientific means of transport and intercourse still threaten us as the most terrible method of mutual extermination, and the nations refuse an obvious resource to common action, common command, or even a common time-table for pacific purposes. Nothing could better signalise the later years of His Majesty's reign, or be more in keeping with the master-spirit of the man whose life we prize and are now commemorating, than the conclusion of such an agreement. It would be backed by all the scientific opinion of the world, and be the most striking proof of the progress of the reign in its essential quality-the pursuit of peace.

\title{
The Structure of the Universe
}

\section{By Sir James Jeans, F.R.s.}

$\mathrm{I}^{\mathrm{N}}$ the last quarter of a century, our picture of the astronomical universe has changed almost beyond recognition, and yet we seem to be standing only on the seashore of the great ocean of knowledge.

The geocentric view of the structure of the universe became untenable for thinking men in the year 1610, but in 1910 many astronomers favoured a 'galacto-centric' view, believing that the galactic system was the central and dominating feature of the astronomical universe, with the earth very near to its geometrical centre.

Sir William Herschel had shown that such stars as he could see in his telescope constituted a coinshaped structure, the more distant stars combining to form the faint band of light we call the Milky Way. In the astronomical language of 1910, a few classes of objects-spiral nebulæ and globular star-clusters-were found to 'shun' this plane, but the majority-irregular and planetary nebulæ, blue and Wolf-Rayet stars, eclipsing and Cepheid variables-'favoured' it, ranging themselves about this plane like flies on the two sides of a fly-paper. For this reason the plane of the Milky Way was thought to be fundamental in the structure of the universe.
So far back as 1755, Kant had shown that other views were possible, suggesting that the elliptical nebulæ were not "enormous single stars, but systems of many stars" similar to our own, but at so vast a distance that their light "on account of their immense multitude, reaches us in a uniform pale glimmer".

Herschel adopted this view, speaking of these supposed other systems of stars as "island universes". It fell into disfavour for a time, but Eddington, writing in 1914, remarked that "the hypothesis has recently been revived as regards the spiral nebulæ". He continued: "It must be admitted that direct evidence is entirely lacking as to whether these bodies are within or without the stellar system".

Then Hubble found it possible to measure the sizes and distances of these objects, and the problem was solved. Certain standard objerts are believed to shine with the same intrinsic luminosity wherever they occur in space, so that their apparent faintness at once gives a measure of their distance. Among such standard beacon-lights are Cepheid variables of assigned period, long-period variables, blue stars of assigned spectral type, and novæ at maximum. Examples of most of these standard 
objects can be detected in the nearer nebulæ, and happily all tell substantially the same story as to the distances of these nebulæ. They tell us that the nearest nebula of all (M 33 in Triangulum) is about 800,000 light-years distant, while the second nearest (M 31, the Great Nebula in Andromeda) is at a distance perhaps about three per cent greater. This latter nebula subtends an angle of about five degrees in the sky, so that its diameter must be about 70,000 light-years. The diameter of our galactic system is generally supposed to be at least three times this.

Such measurements and studies have made it clear that these nebulæ are systems of stars like our own galaxy, that they lie entirely clear of this and are substantially smaller than it is. If we represent our own galaxy by London, then Birmingham and Bristol will represent the two nearest external galaxies fairly well in respect of both size and distance. A small nebula (M 32) which accompanies the Great Nebula in Andromeda may be represented by Wolverhampton or Coventry, Also two minor star-systems, the Magellanic Clouds, which lie so near our own galaxy (90,000 light-years from the sun) as almost to form part of it, may perhaps be compared to Croydon and Sutton. We must not place our sun in Central London, as Herschel imagined; rather we are out at Hampstead or Highgate, and see the lights of Central London and the smoky pall over it in the distance, when we look towards the great star-clouds and dark nebulæ of Sagittarius.

Most of these nebulæ show the same flattened shape as our own galaxy, and it has long been conjectured that this flattening must indicate rotation. Recently rotation has been discovered spectroscopically in a number of the nebulæ. The central part of the Great Nebula in Andromeda, for example, rotates with a period of about 16 million years, while that of the well-known nebula N.G.C. 4594 in Virgo rotates about twice as fast. Quite recently Oort, Plaskett, Lindblad and others have found that the galactic system is also in rotation. The stars revolve much like the planets or the particles of Saturn's rings, the period of revolution increasing as we pass outwards. At the sun's distance it is at least 200 million years. This is $12 \frac{1}{2}$ times the period of revolution just mentioned for the Andromeda nebula, but it refers to a point six times as far out. If the whole of this latter nebula were concentrated in or near its centre, the rotation period at the sun's distance out would be about 235 million years, so that the rotations are at least comparable.

From these rotation periods, it is of course possible to calculate the masses of the nebulæ and of the galactic system. The nebulæ are found to have the masses of thousands of millions of suns, while the galaxy has a mass of 100,000 million suns at least. We see that our galaxy is something of a giant in mass as well as in size ; if the nebulæ are island-universes, we still inhabit a continent.

As we proceed outwards into space, the Cepheid variables and other standard beacon-lights so far mentioned sink one after another into invisibility. Hubble has, however, found that nebulæ of assigned shapes and structure are themselves standard articles to a reasonably good approximation. Thus the faintness of the nebulæ themselves gives a measure of their distance, and it becomes possible to estimate the distances of even the faintest nebulæ, right up to the limits of vision of the telescope. The nebulæ prove to be distributed fairly uniformly at an average distance apart of perhaps 1,800,000 light-years.

If the matter contained in all these nebulæ were scattered evenly through space, the density would be of the order of $10^{-30}$ grams per c.c. This may give a clue to the mode of formation of the nebulæ, since a gas of this density would tend to condense into 'droplets' of just about the observed masses of the nebulæ. If nebulæ originated as such condensations in a fairly uniform gas, we have a ready explanation of the comparative uniformity of their sizes and structure.

When the light from any one of these distant nebulæ is analysed spectroscopically, the whole spectrum is found to be displaced homologously towards the red end. If we interpret these spectral displacements in the simplest way, as pure Dopplereffects, then these nebulæ are found to be receding from our galaxy at speeds almost exactly proportional to their distances-roughly, 105 miles a second for each million light-years of distance-and when allowance is made for the sun's motion through the galaxy, the same is found to be true of the nearer nebulæ also. In brief, the whole universe appears to be expanding uniformly, its linear dimensions increasing by one per cent every 20 million years.

It is likely that this apparent recession of the nebulæ is something more than a mere astronomical phenomenon, for the generalised theory of relativity seems to call for an expansion (or alternatively a contraction) of space itself. Thus the motions of the nebulæ may well be indications of something far more fundamental-a uniform expansion of the space in which they are imbedded.

The theory of relativity associates gravitation with a curvature of the space-time continuum; this is curled up in the proximity of matter, and the curvature shows itself in the curved paths of planets and projectiles. At one time it scarcely seemed possible that the whole curvature of the space-time continuum could be of this kind, for analysis showed that, if it were, space could 
not stand still ; it would either expand or contract. To avoid this apparent absurdity, Einstein imagined the continuum endowed with a further curvature of its own, independent of the presence of matter and so inherent in the space itself. This was specified by a quantity, the 'cosmical constant', which was supposed to have a uniform value everywhere and so kept the total volume of space fixed and unalterable.

There is no observational evidence that such a constant exists, for the curvature it implies is too small for measurement. The constant was only introduced because Einstein had thought space must be at rest, and there is no need to retain it now that space appears not to be at rest. On the other hand, we are under no compulsion to discard it. Actually Einstein and de Sitter have found that the constant can have a large range of values, including zero, without running counter to any of the observed facts of astronomy.

We may compare space-time to a river having space as its cross-section and time as the direction of flow of its stream. Two dimensions are, of course, missing; the cross-section of our river ought to have three dimensions instead of one, but as all three are all exactly similar, the suppression of two of them does no great harm.

If space could remain constant in size, this river would become a canal with parallel banks; Einstein's original space-time river was of this type. But Friedmann and Lemaître showed that such a space would be unstable; any slight disturbance or irregularity-such as would, for instance, be caused by the condensation of a primeval gas into nebulæ-would start it either expanding or contracting. For this reason Lemaître thought that the Einstein canal should be replaced by a sort of Amazon River, starting from minute beginnings and for ever widening as it flows-expanding space. De Sitter found that other values for the cosmical constant made two other types of solution mathematically possible. In one of these the canal-like river gives place to a sort of Panama Canal - space first contracts until it reaches a minimum and then expands again to an indefinite extent. In the other, space rhythmically expands and contracts, so that the spacetime river becomes a series of regularly spaced lakes connected by narrows.

The Amazon-like space-time river of Lemaître was open to one grave objection. Its length, which is time-the whole time since the beginning of the universe-was limited, and its source was nothing like distant enough to allow for the observed stages of development of stellar systems -in brief, the stars were too old to have grown up within the length of the river.

The two more recent solutions of de Sitter and Einstein are not open to any such objection, and at present either of them appears capable of providing a true, although highly artificial, representation of the observed phenomena of the universe. At one time, de Sitter was advocating the Panama canal type of map, while Einstein favoured the rhythmical universe of lake and narrows-a space which alternately expanded and contracted. Einstein now appears to contemplate the possibility of a zero cosmical constant and a space of infinite extent. But it is, I think, fair to say that no one is satisfied with the present position. It may be that still other alternatives remain to be discovered, and another few years may witness some new formulation of the problem which will lead to a satisfactory solution.

\section{The New Age in Physics}

\section{By Dr. H. Dingle, Imperial College of Science and Technology, London}

$\mathrm{E}^{\mathrm{n}}$ VERY advance in thought has two aspects - the loss of the old and the gain of the new-and it is probably inevitable that, after the first flush of excitement has faded away, the former should become the more conspicuous. It may inspire joy at the passing of a delusion, or regret at the failure of an ideal : in either case it is the negative aspect of the change which protrudes itself, because all are conscious that what they believed in has gone, but only a few can at first see the significance of the new thing which has come.

This is exemplified by the fact, which is in all our minds to-day, that King George V has occupied the throne of England for twenty-five years. What does it mean? In 1910 we knew well enough what it would mean; but in 1935, who except a mathematical physicist will commit himself to an opinion? Twenty-five years to one observer, we are told, may be fifty years to another, and neither can claim superiority for his time-scale. Why, then, not celebrate a golden instead of a silver jubilee? The relativist knows, of course, that the destruction of absolute time is merely the necessary preliminary to the building of an absolute 'interval', and that twenty-five years is the interval during which King George has reigned. In this matter His Majesty's time is proper time, so that physics 